Proceedings of the 15th Czech and Slovak Conference on Magnetism, Košice, Slovakia, June 17-21 2013

\title{
High Resolution Tips for Switching Magnetization MFM
}

\author{
M. PrecneR*, J. Fedor, J. TóBik, J. ŠoltÝs, V. CAmbel
}

Institute of Electrical Engineering, Slovak academy of Sciences, Bratislava, 84104 Slovak Republic

\begin{abstract}
Switching magnetization magnetic force microscopy (SM-MFM) is based on two-pass magnetic force microscopy with opposite orientation of tip magnetization between two scans. The sum of the scanned data with reversed tip magnetization depicts local van der Waals forces, and their difference maps the local magnetic forces. Tip magnetization can be easily reversed in external magnetic field during the scanning. The separation of the forces mapped enables scanning in close proximity of the sample $(\sim 5 \mathrm{~nm})$. Therefore, extremely high spatial resolution $(10 \mathrm{~nm})$ is achievable by the SM-MFM. Image phase resolution of the MFM method depends on various geometric parameters of the tip, such as tip length, its apex radius and taper angle. The parameters are determined by the evaporation process, within which the standard atomic force microscopy tips are coated with magnetic layer. In this work we show that the thickness of the coated layer is important for the SM-MFM spatial resolution.
\end{abstract}

DOI: $10.12693 /$ APhysPolA.126.386

PACS: 75.75.+a, 75.70.-i, 68.37.Rt, 75.60.Jk, 81.15.Ef, 02.30.Nw

\section{Introduction}

Novel magnetic memories require higher integration bit density. Bit pattern media (BPM) with a bit size close to $10 \mathrm{~nm}$ have been achieved and are a leading topic of the developed new magnetic storage media [1]. Magnetic force microscopy (MFM) is a famous method for investigation of domains in magnetic samples for a samplesurface preparation and relatively high spatial resolution $(\sim 30 \mathrm{~nm})$. However, the resolution is not satisfactory for developed magnetic memories.

MFM is a two-pass technique, therefore in the first step topography is measured in contact or semicontact mode. In the second pass the tip is lifted to a constant height (more than $20 \mathrm{~nm}$ ) to eliminate van der Waals forces and to evaluate a phase shift which corresponds to a magnetic tip-sample interaction [2].

Several groups try to improve the MFM spatial resolution by developing sharp and high aspect-ratio tips, or by special deposition conditions that use seed layers to achieve a perpendicular magnetic anisotropy [3-5]. But, MFM spatial resolution is limited by the principle of the two pass technique, for which the tip-sample separation has to be more than $20 \mathrm{~nm}$ to guarantee that magnetic forces dominate the van der Waals forces.

In the two-pass method developed, the so-called switching magnetization MFM (SM-MFM), we use low momentum magnetic tips and evaluate phase shift at the tip-sample distance of $5 \mathrm{~nm}$. Two phase images are taken, the second with reversed magnetization of the tip (Fig. 1). Then, the sum of the two signals gives van der Waals and electrostatic forces, and from their difference we get clear magnetic forces. Tip magnetization is switched by external magnetic field applied in parallel with the sample surface [6-8]. The method we propose eliminates drawbacks of standard MFM method and is a favourable technique for improving the MFM spatial resolution below $10 \mathrm{~nm}$.

*corresponding author; e-mail: marian.precner@savba.sk

\section{Experimental results and discussion}

We have prepared magnetic tips in a magnetic single domain state for the SM-MFM technique. The state can be reversed by external magnetic field oriented in parallel with the sample. One of the most important parameters that influence the MFM spatial resolution is the thickness of the magnetic layer located on the tip. In our case, Permalloy $\left(\mathrm{Ni}_{81} \mathrm{Fe}_{19} ; \mathrm{Py}\right)$ was evaporated onto one of the tip's sidewalls (tip NSG01 [9]) under the specific angle to create $\mathrm{Py}$ triangle in a single domain state. We use $5 \mathrm{~nm} \mathrm{Au}$ as a seed layer for a better adhesion and elimination of the stress between Py layer and the tip. Py was deposited by e-beam evaporation at a base vacuum of $5 \times 10^{-8}$ Torr, and the grow rate was fixed at $1 \AA / \mathrm{s}$ for 5 different thicknesses $(6,12,18,24,30 \mathrm{~nm})$. The SM-MFM resolution was evaluated in the scanning system NTegra (produced by NT-MDT co.) which has implemented in-plane magnetic field used to reverse the tip magnetization.

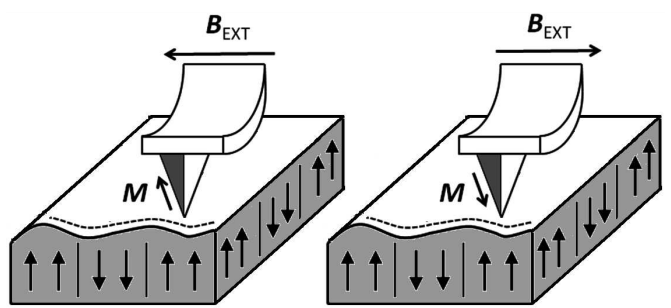

Fig. 1. Principle of SM-MFM technique with reversal tip magnetization (arrow close to the tip) at low lift distance (below $5 \mathrm{~nm}$ ) provided by external magnetic field $\boldsymbol{B}_{\text {ext }}$ between two scans.

In order to evaluate spatial resolution we use the fast Fourier transform (FFT) analysis [10]. According Ref. 10, the spatial resolution corresponds to the information limit - a frequency, for which the signal dominates white noise, which is represented by the knee of the FFT signal. Spatial resolution of the SM-MFM tips was demonstrated on a perpendicular magnetic recording media (PMR) with a bit density of $625 \mathrm{Gbit} /$ inch $^{2}$. Fig- 
ure 2a shows a typical topography (roughness $\sim 1 \mathrm{~nm}$ ) and Fig. 2b-2f show phase images of PMR media taken by tips that differ in a Py layer thickness $(6,12,18,24$ and $30 \mathrm{~nm}$, respectively). FFT (Fig. 3) shows the spatial resolution of the phase image from Fig. 2. SM-MFM tips with Py layer thickness of $12 \mathrm{~nm}$ (c) and $18 \mathrm{~nm}$ (d) show the best spatial resolution of $10 \mathrm{~nm}$ and $11 \mathrm{~nm}$, respectively (arrows inside indicate information limit knee), calculated as one half of $\lambda_{k n e e}$. Spatial resolution is worse $(13,14,16 \mathrm{~nm})$ for Py thicknesses $6,24,30 \mathrm{~nm}$, respectively. The low-frequency signal (Fig. 3), as well as magn. moment of SM-MFM tips, increase with increasing Py thickness.
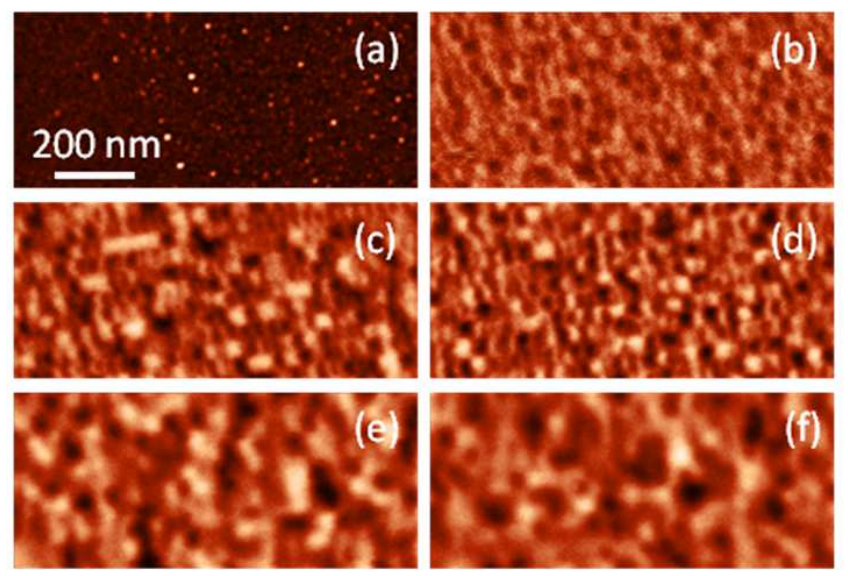

Fig. 2. Topography (a) and MFM phase images (b, c, d, e, f) a PMR medium $\left(625 \mathrm{Gbit} /\right.$ inch $\left.^{2}\right)$ written at a $5 \mathrm{~nm}$ tip-sample separation. Thicknesses of the Py layers are $6 \mathrm{~nm}$ (b), $12 \mathrm{~nm}$ (c), $18 \mathrm{~nm}(\mathrm{~d}), 24 \mathrm{~nm}(\mathrm{e})$, and $30 \mathrm{~nm}(\mathbf{f})$.

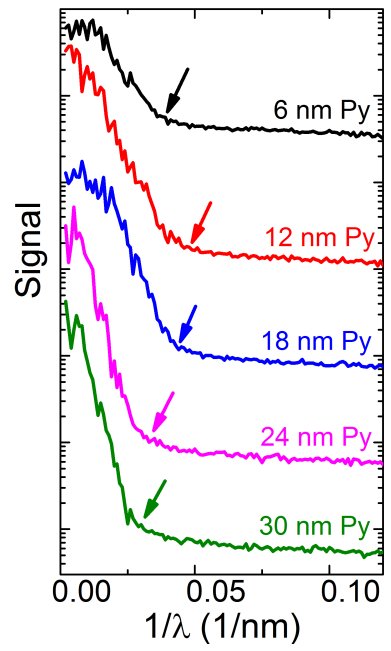

Fig. 3. FFT of the bit track from Fig. 2 for different Py layers. Spatial resolution is calculated as a half of $\lambda$ and it is shown with arrows. Curves were shifted along $\mathrm{y}$-axis by multiplying $\mathrm{y}$-range for better illustration.

When the lift height is below $20 \mathrm{~nm}$, van der Waals forces start to dominate over magnetic forces.In the SMMFM method two scans in a lift height of $5 \mathrm{~nm}$ were taken with positive (Fig. 4a) and negative (Fig. 4b) magnetization of the tip, then the difference gives clear magnetic forces (4c) and their sum gives van der Waals forces at the height of $5 \mathrm{~nm}(4 \mathrm{~d})$.
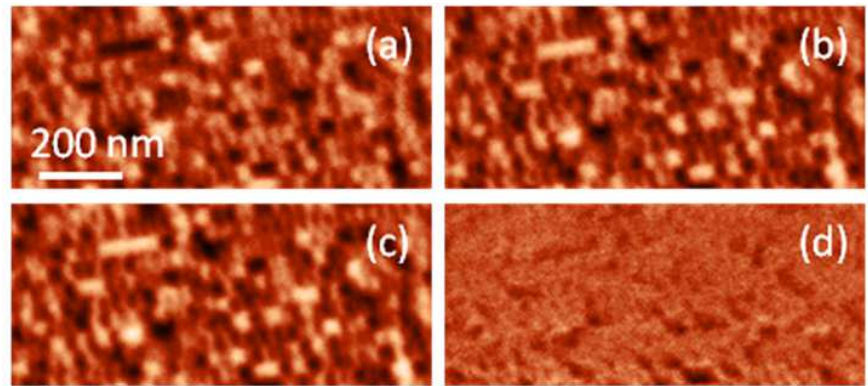

Fig. 4. Phase image from perpendicular magnetic media are taken with positive (a) and negative (b) magnetization of SM-MFM tip at lift height of $5 \mathrm{~nm}$. Magnetic forces $(c)=(a)-(b)$ and van der Waals forces (topography) $(\mathrm{d})=(\mathrm{a})+(\mathrm{b})$ are calculated.

\section{Conclusions}

In this work we have optimized a magnetic layer thickness for the SM-MFM tips. For Py layer thickness $12 \mathrm{~nm}$, a spatial resolution of $10 \mathrm{~nm}$ was achieved. Moreover, the SM-MFM technique separates van der Waals forces, electrostatic forces and magnetic forces and allows magnetic field mapping in close proximity to the sample.

\section{Acknowledgments}

This work was supported by Slovak Grant Agency APVV, projects APVV-0088-12 and VVCE-0058-07, by VEGA project $2 / 0037 / 12$, and by the Research \&Development Operational Program funded by the ERDF, "CENTE", ITMS code 26240120011(0.5).

\section{References}

[1] O. Mosendz, S. Pisana, J.W. Reiner, B. Stipe, D. Weller, J. Appl. Phys. 111, $07 \mathrm{~B} 729$ (2012).

[2] http://www.ntmdt.com/spm-principles/view/ ac-mfm.

[3] M.R. Koblischkaa, U. Hartmanna, T. Sulzbach, J. Magnetism Magn. Mater. 272-276, 2138 (2004).

[4] P.B. Fischer, M.S. Wei, S.Y. Chou, J. Vac. Sci. Technol. B 11, 2570 (1993).

[5] A. Schwarz, R. Wiesendanger, Nanotoday 3, 28 (2008).

[6] V. Cambel, P. Eliáš, D. Gregušová, J. Fedor, J. Martaus, G. Karapetrov, V. Novosad, I. Kostič, J. Nanosci Nanotechnol 10, 4477 (2010).

[7] V. Cambel, P. Eliáš, D. Gregušová, J. Martaus, J. Fedor, G. Karapetrov, V. Novosad, J. Magnetism Magn. Mater. 322, 2715 (2010).

[8] V. Cambel, M. Precner, J. Fedor, J. Šoltýs, J. Tóbik, T. Ščepka, G. Karapetrov, Appl. Phys. Lett. 102 , 062405 (2012).

[9] http://www.ntmdt-tips.com/products/view/ nsg01.

[10] S.V. Kalinin, S. Jesse, B.J. Rodriguez, J. Shin, A.P. Baddorf, H.N. Lee, A. Borisevich, S.J. Pennycook, Nanotechnology 17, 3400 (2006). 